\title{
Differential prognostic accuracy of right ventricular dysfunction, the Seattle heart failure model and the MAGGIC score in patients with severe mitral regurgitation undergoing the MitraClip ${ }^{\circledR}$ procedure
}

\author{
S. Heyl, B. Luu, M. Wieszner, A. Nikkhoo, F. Seeger, K. Hemmann, B. Assmus, B. Kaess, A.M. Zeiher, \\ C. Walther, S. Fichtlscherer, J. Honold* \\ University Hospital Frankfurt, Department of Cardiology, Germany
}

\section{A R T I C L E I N F O}

\section{Article history:}

Received 30 June 2020

Received in revised form 29 August 2020

Accepted 3 September 2020

Available online 12 October 2020

\section{Keywords:}

MitraClip

Seattle heart failure model

MAGGIC score

TAPSE

Functional mitral regurgitation

\begin{abstract}
A B S T R A C T
Background: MitraClip ${ }^{\circledR}(\mathrm{MC})$ is an established procedure for severe mitral regurgitation (MR) in patients deemed unsuitable for surgery.

Right ventricular dysfunction (RVD) is associated with a higher mortality risk. The prognostic accuracy of heart failure risk scores like the Seattle heart failure model (SHFM) and Meta-Analysis Global Group in Chronic Heart Failure (MAGGIC) score in pts undergoing MC with or without RVD has not been investigated so far.

Methods: SHFM and MAGGIC score were calculated retrospectively. RVD was determined as tricuspid annular plane systolic excursion (TAPSE) $\leq 15 \mathrm{~mm}$. Area under receiver operating curves (AUROC) of SHFM and MAGGIC were performed for one-year all-cause mortality after MC.

Results: $\mathrm{N}=103$ pts with $\mathrm{MR} \mathrm{III}^{\circ}(73 \pm 11$ years, $\operatorname{LVEF} 37 \pm 17 \%)$ underwent $\mathrm{MC}$ with a reduction of at least $\mathrm{I}^{\circ}$ MR. One-year mortality was $28.2 \%$.

In Kaplan-Meier analysis, one- year mortality was significantly higher in RVD-pts (34.8\% vs 2.8\%, $\mathrm{p}=0.009$ ).

Area under the Receiver Operating Characteristic (AUROC) for SHFM and MAGGIC were comparable for both scores (SHFM: 0.704, MAGGIC: 0.692). In pts without RVD, SHFM displayed a higher AUROC and therefore better diagnostic accuracy (SHFM: 0.776; MAGGIC: 0.551, p < 0.05). In pts with RVD, MAGGIC and SHFM displayed comparable AUROCs.

Conclusion: RVD is an important prognostic marker in pts undergoing MC. SHFM and MAGGIC displayed adequate over-all prognostic power in these pts. Accuracy differed in pts with and without RVD, indicating higher predictive power of the SHFM score in pts without RVD.
\end{abstract}

(C) 2020 Published by Elsevier B.V. This is an open access article under the CC BY-NC-ND license (http:// creativecommons.org/licenses/by-nc-nd/4.0/).

\section{Introduction}

The transcatheter mitral valve edge-to edge repair (TMVR) using the MitraClip device (Abbott, USA) has emerged as therapeutic tool for patients with severe symptomatic mitral regurgitation $\left(\mathrm{MR} \mathrm{III}{ }^{\circ}\right.$ ) who are deemed unsuitable for mitral valve surgery by the heart team.

In pivotal trials, TMVR showed similar improvements in clinical outcomes with a superior safety profile compared to conventional surgery in these patients [1].

\footnotetext{
* Corresponding author at: University Hospital Frankfurt, Department of Cardiology, Theodor-Stern-Kai 7, 60590 Frankfurt, Germany.

E-mail address: joerg.honold@kgu.de (J. Honold).
}

Several large registries have since demonstrated sustained clinical benefit and echocardiographic improvement of MR severity after the procedure [2-4].

Consensus exists that the presence of mitral regurgitation has a negative impact on prognosis in heart failure patients [5,6]. Moreover, in the COAPT trial, patients with moderate to severe functional MR were randomized to either optimal medical treatment (OMT) alone or additional TMVR [7]. Patients in the interventional group demonstrated lower mortality and fewer heart failure hospitalizations, which held up during long term follow up [8]. However, the Mitra FR trial failed to show a mortality benefit at twelve months [9].

A number of important comorbidities and pathophysiological conditions have been described to attenuate the beneficial effect of TMVR in these selected multimorbid patients, such as depressed 
left atrial systolic function, atrial fibrillation and pulmonary hypertension [10-12]. Proper identification of patients likely to benefit from the procedure would therefore be highly advantageous.

In heart failure patients, prognostic models like the Seattle Heart Failure Model (SHFM) and the Meta-Analysis Global Group in Chronic (MAGGIC) Heart Failure score are widely used [1316]. However, only limited data exist about the prognostic accuracy of these scores in patients referred for TMVR [17]. Moreover, the prognostic accuracy of these scores in the context of possible interactions with important determinants of prognosis in patients suffering from $\mathrm{MR} \mathrm{III}{ }^{\circ}$ such as right heart dysfunction has not been separately described so far in the setting of TMVR.

Therefore, aim of the present study was to describe the prognostic utility of the SHFM and the MAGGIC score in patients with severe mitral regurgitation after TMVR with and without right ventricular dysfunction, as assessed by tricuspid annular plane excursion (TAPSE).

\section{Methods}

A total number of 103 consecutive patients were included in this retrospective observational analysis after TMVR in our institution. All patients gave their written informed consent to collect, analyze and publish patient-related data prior to data analysis. The study was approved by the local ethics committee (protocol $\mathrm{Nr}$. 512/15) and performed in accordance with the declaration of Helsinki.

\subsection{Heart team}

As recommended by the ESC guidelines [17], every patient was discussed in detail by a heart team composed of interventional and noninterventional cardiologists, cardiac surgeons and anesthesiologists prior to therapy. If relevant for the individual patient, physicians of other specialties were also consulted. Factors favoring TMVR over a surgical approach included the patient's comorbidities, age, frailty, previous cardiac surgery, anatomy amenable to TMVR and high surgical risk (as determined by the EUROScore II) [18].

\subsection{TMVR procedure}

All patients received TMVR by the MitraClip device under general anesthesia, guided by both fluoroscopy and transesophageal echocardiography as previously described [1]. After femoral transvenous access and atrial transseptal puncture, the device is aligned with the regurgitant jet. Using the delivery system, the arms of the device are opened to grasp and approximate the leaflets. In case of inadequate MR reduction, the device may be moved, or additional Clips may be placed.

\subsection{Echocardiography}

Comprehensive transthoracic and transesophageal echocardiography was performed before the procedure. Transthoracic echocardiography was done during follow up according to the recommendations of the European Society of Cardiology [19]. The examinations included an assessment of severity, mechanism and suitability for interventional edge- to- edge repair.

Tricuspid annular plane systolic excursion (TAPSE) was measured before TMVR using the lateral tricuspid annulus from the apical 4- chamber view with an M- mode cursor [20,21]. A TAPSE $\leq 15 \mathrm{~mm}$ was defined as RVD [22].

Pulmonary artery systolic pressure was estimated using the peak tricuspid regurgitation velocity added to the right atrial pres- sure which was estimated based on size and collapsibility of the inferior vena cava [23].

\subsection{Follow- up and outcome}

Follow up was performed by phone calls with the patientś relatives, family physicians, or contacting the local registry office authorities about the patientś vital status.

\subsection{Prediction of survival by the Seattle heart failure model}

The Seattle Heart Failure Model (SHFM) integrates demographic characteristics, clinical features, laboratory values as well as implemented pharmacological and device treatments to comprehensively asses a heart failure patient's prognosis [24]. It was originally derived in a clinical trial cohort of HF patients with reduced left ventricular ejection fraction (LVEF) $<35 \%$ and severe symptoms (NYHA class IIIB or IV) with external validation in additional HF cohorts from clinical trials and outpatient settings [25]. It has been used as a predictor of mortality, LVAD placement and to evaluate the benefit of cardiac devices [26].

SHFM scores were calculated using the SHFM webpage (https:// depts.washington.edu/shfm).

\subsection{Prediction of survival by the MAGGIC score}

The Meta- Analysis Global Group in Chronic Heart Failure (MAGGIC) mortality risk model contains the following predictor variables: age, sex, body mass index, systolic blood pressure, LVEF, creatinine, current smoker, diabetes mellitus, chronic obstructive pulmonary disease, New York Heart Association (NYHA) class, HF duration $>18$ months, beta -blocker use and angiotensinconverting enzyme inhibitor use [27]. It includes patients from 30 cohort studies and includes both heart failure patients with reduced and preserved ejection fraction. It has been shown to predict all-cause mortality in patients undergoing transcatheter aortic valve replacement [13].

MAGGIC scores were calculated using the online calculator (https://www.mdcalc.com/maggic-risk-calculator-heart-failure).

\subsection{Statistical analysis}

Statistical analysis was performed using SPSS Version 23.0 for Windows. Categorical variables were expressed as counts and percentages, continuous variables with normal distribution as mean \pm SD or median and interquartile range after testing for normal distribution. Categorical variables were analyzed by chisquare testing. For continuous variables, the Mann Whitney $U$ test was performed.

The Kaplan- Meier method was used to chart event rates, survival curves were compared using the log- rank test.

Using C- statistics (“concordance”), specifically Delong's test for two correlated ROC curves, the receiver operating curves of both scores were compared to differentiate the discriminative ability of the MAGGIC and SHFM scores. The Software R Version 3.5.2 by Revolution Analytics was employed for this purpose.

Multivariate analysis for one-year all-cause mortality was performed using the Cox proportional hazard model.

All testing was performed with two-sided $\mathrm{p}<0.05$ as level of significance. 


\section{Results}

\subsection{Baseline characteristics and procedure}

Between June 2013 and February 2017, a total number of 103 patients underwent successful TMVR with the MitraClip device in our institution after consensus for TMVR approach by the interdisciplinary heart team. All patients reported heart failure symptoms despite optimal medical treatment according to contemporary guidelines for pharmacotherapy in heart failure at the time of TMVR [19,28].

Right ventricular dysfunction defined as a TAPSE of $15 \mathrm{~mm}$ or less was present in 46 (44.6\%) of the 103 patients. Patients with RVD demonstrated significantly lower left ventricular ejection fraction and higher NT-proBNP serum levels. Consecutively, mean EuroScore II, MAGGIC Score and SHFM score were higher in these patients (see Table1). Baseline characteristics are depicted in Table 1.

Degenerative MR was the underlying etiology in 60 (58.3\%) of the patients, functional MR in 43 (41.7\%) patients. The etiology of MR did not differ significantly between patients with and without RVD, with a higher proportion of degenerative MR in the group with preserved right ventricular function $(30.1 \%$ versus $28.2 \%$ in patients with TVD, $\mathrm{p}=0.17$ ).

Compared to patients with degenerative MR, patients with FMR were significantly more often male ( 81.4 vs $63.3 \%, p=0.047$ ), demonstrated more often HFrEF with severely reduced LVEF (mean LVEF $29.8 \pm 12.2 \%$ versus $41.4 \pm 18.9 \%, \mathrm{p}=0.001$ ) with more pronounced LV remodeling (left ventricular end-diastolic diameter $64.5 \pm 10.1 \mathrm{~mm}$ vs $59.5 \pm 10.2 \mathrm{~mm}, \mathrm{p}=0.028)$. Mean TAPSE did not differ between groups (FMR: $16.3 \pm 4.2$ versus DMR: $17.1 \pm 3.9, \mathrm{p}=0.3)$. RVD defined as TAPSE $<15 \mathrm{~mm}$ was present in $28(65.1 \%)$ of FMR-patients and 31 (51.7\%) of DMR-patients, $\mathrm{p}=0.12$.

During the TMVR procedure, a median number of 2 clips (interquartile range (IQR) 1) were implanted. After therapy, a reduction of MR to MR grade 0 or 1 was achieved in 74 (71.8\%) patients, 25 pts (24.3\%) had residual MR II, 4 (3.9\%) pts MR had residual moderate to severe MR.

Mean duration of follow up was $11.7 \pm 11.5$ months. No patient was lost to follow up. Within one year after TMVR, a total number of 29 patients died, accounting for an all-cause mortality rate of $28.2 \%$.

\subsection{Prognostic value of right ventricular dysfunction after TMVR}

Fig. 1 shows the survival curves of patients with and without RVD, with a significantly higher one-year mortality in patients with RVD present at the time of therapy of $34.8 \%$ compared to $22.8 \%$ in patients without RVD.

To identify the prognostic impact of RVD in the settings of functional and degenerative MR, separate Kaplan Meier survival analyses were applied for the subsets of patients with predominantly functional or degenerative etiology of mitral regurgitation:

Fig. 2 depicts the one-year survival for patients with FMR stratified according to their right ventricular function: FMR-patients with a TAPSE $\leq 15 \mathrm{~mm}$ had a significantly higher all- cause mortality one year after TMVR (38.1\%) compared to FMR-patients with preserved right ventricular function (9.1\%).

In contrast, in patients with degenerative MR, Kaplan Meier estimates did not differ significantly between the subgroups of DMR-patients with preserved or reduced right ventricular function (32\% one-year mortality in DMR-patients with RVD versus $34.3 \%$ in DMR-patients without RVD), see Fig. 3.

\subsection{Differential prognostic utility of the SHFM and MAGGIC score dependent on the presence of RVD}

Retrospective application of the SHFM and MAGGIC score revealed moderate over-all sensitivity and specificity for prediction of one-year all-cause mortality in the whole patients collective, with an area under the receiver operating curve value of 0.704 for the SHFM score and 0.692 for the MAGGIC score. Fig. 4 repre-

Table 1

Baseline Characteristics. In case of missing values, the number of available values is denoted in parentheses (n/N). Values are mean \pm SD or $n$ (\%) unless otherwise specified.

\begin{tabular}{|c|c|c|c|c|}
\hline & Total $(\mathrm{N}=103)$ & TAPSE $<=15 \mathrm{~mm}(\mathrm{~N}=46)$ & TAPSE $>15 \mathrm{~mm}(\mathrm{~N}=57)$ & $\mathrm{p}$ \\
\hline Age - y & $72.8 \pm 10.84$ & $72 \pm 11.37$ & $73.8 \pm 10.84$ & 0.396 \\
\hline Male sex -n (\%) & $73(70.9)$ & $34(33)$ & $39(37.9)$ & 0.349 \\
\hline Diabetes $-\mathrm{n} .(\%)$ & $38(36.9)$ & $23(22.3)$ & $15(14.6)$ & 0.611 \\
\hline Hypertension -n. (\%) & $81(78.6)$ & $36(35)$ & $45(43.7)$ & 0.497 \\
\hline Creatinine clearance $(\mathrm{ml} / \mathrm{min})$ & $53.3 \pm 22$ & $50.6 \pm 21$ & $56.8 \pm 23.1$ & 0.165 \\
\hline Median NTproBNP (ng/l) (IQR) (83/103) & $2540(1230-4204)$ & $2989(1583-5361)$ & $1639(898,3-3790,8)$ & 0.014 \\
\hline \multicolumn{5}{|l|}{ Medication use } \\
\hline Antiplatelet agents (100/103) & $51(49.5)$ & $22(47.8)$ & $29(50.9)$ & 0.859 \\
\hline ACEI/ ARB $(100 / 103)$ & $91(88.3)$ & $37(80.4)$ & $54(94.7)$ & 0.032 \\
\hline Beta blocker (100/103) & $85(82.5)$ & $38(82.6)$ & $47(82.5)$ & 0.735 \\
\hline MCRA $(100 / 103)$ & $51(49.5)$ & $26(56.5)$ & $25(43.9)$ & 0.151 \\
\hline Diuretic drugs (other than MCRA) (100/103) & $87(84.5)$ & $41(89.1)$ & $46(80.7)$ & 0.103 \\
\hline NYHA class - n./ total n. $(\%)$ & & & & 0.462 \\
\hline II & $20(19.4)$ & $7(6.8)$ & $13(12.6)$ & \\
\hline III & $74(71.8)$ & $34(33)$ & $40(38.8)$ & \\
\hline IV & $9(8.7)$ & $5(4.9)$ & $4(3.9)$ & \\
\hline Left ventricular ejection fraction (\%) & $36.51 \pm 17.31$ & $32.4 \pm 16.25$ & $42.2 \pm 17.32$ & 0.004 \\
\hline LVEDV - ml (99/103) & $191.7 \pm 82.36$ & $196.1 \pm 79.4$ & $185.5+-87$ & 0.53 \\
\hline EROA, PISA $\left(\mathrm{mm}^{2}\right)(91 / 103)$ & $42.15(14.32)$ & $42.13 \pm 14.36$ & $42.16 \pm 14.46$ & 0.992 \\
\hline TAPSE $(\mathrm{mm})$ & $16.74 \pm 4.08$ & $13.28 \pm 1.76$ & $19.53 \pm 3.03$ & $<0.001$ \\
\hline Right ventricular systolic pressure $(\mathrm{mmHg})(80 / 103)$ & $52.86 \pm 15.31$ & $50.27 \pm 15.58$ & $56.19 \pm 14.5$ & 0.86 \\
\hline Degenerative MR (\%) & $60(58.3)$ & $29(28.2)$ & $31(30.1)$ & 0.17 \\
\hline Median EuroSCORE II (IQR) & $6.75(3-11.9)$ & $9.19(4.84-15)$ & $4.24(2.47-7.36)$ & $<0.001$ \\
\hline MAGGIC 1-year mortality (\%) & $23.77 \pm 11.38$ & $25.8+-12.47$ & $21 \pm 9.2$ & 0.033 \\
\hline SHFM 1-year mortality (\%) & $83.74 \pm 15.9$ & $81.3 \pm 19.2$ & $87.1 \pm 9.21$ & 0.07 \\
\hline
\end{tabular}

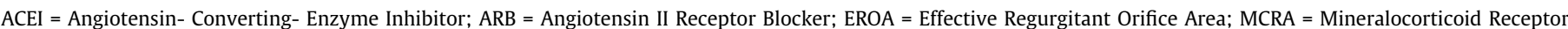

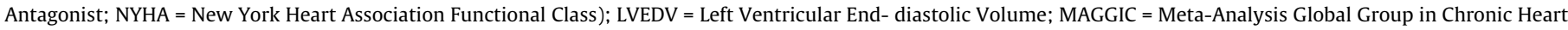
Failure; PISA = Proximal Isovelocity Surface Area; SHFM = Seattle Heart Failure Model; TAPSE = Tricuspid Annular Plane Excursion; 


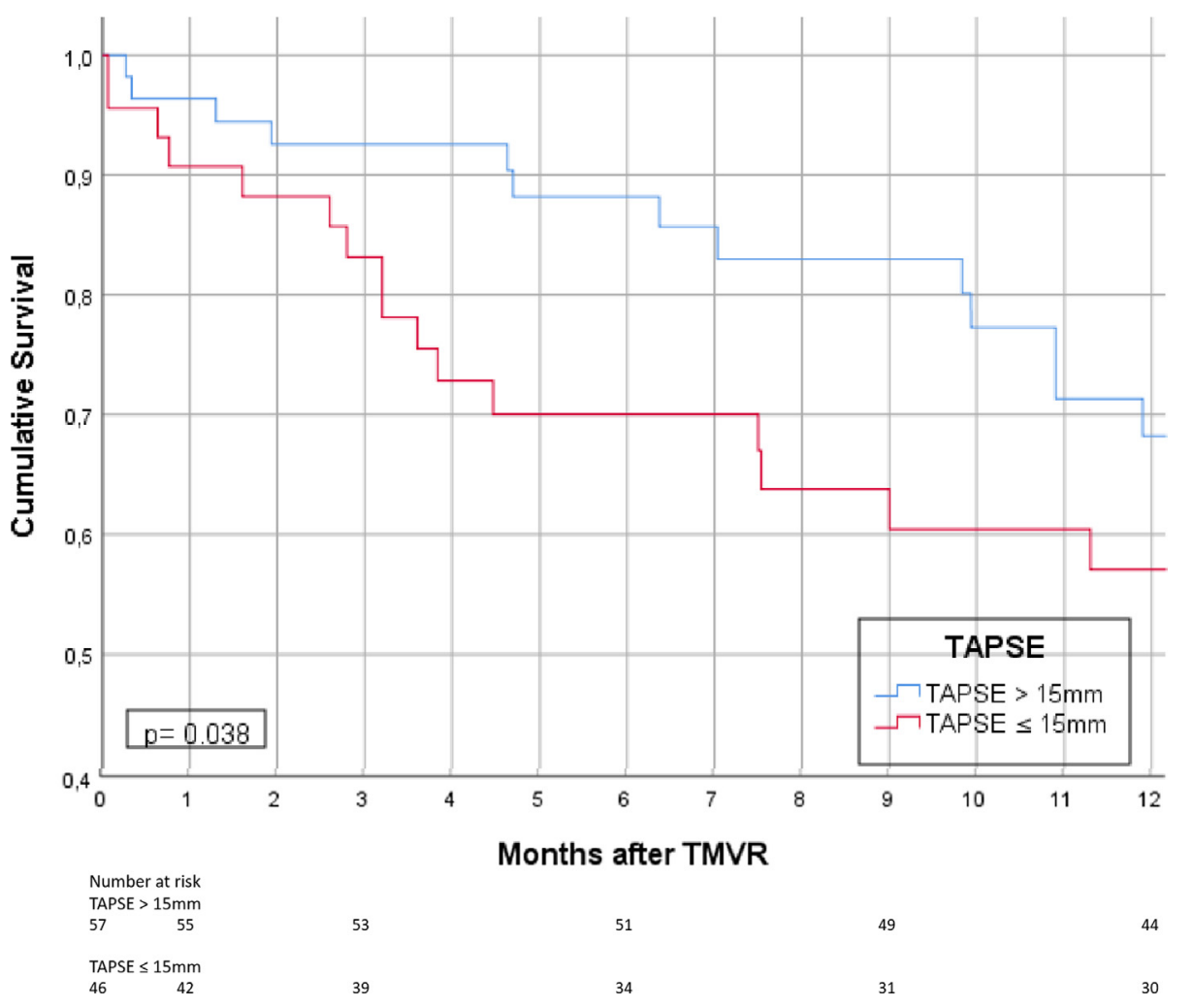

Fig. 1. Kaplan-Meier survival estimates according to tricuspid annular plane systolic excursion (TAPSE).

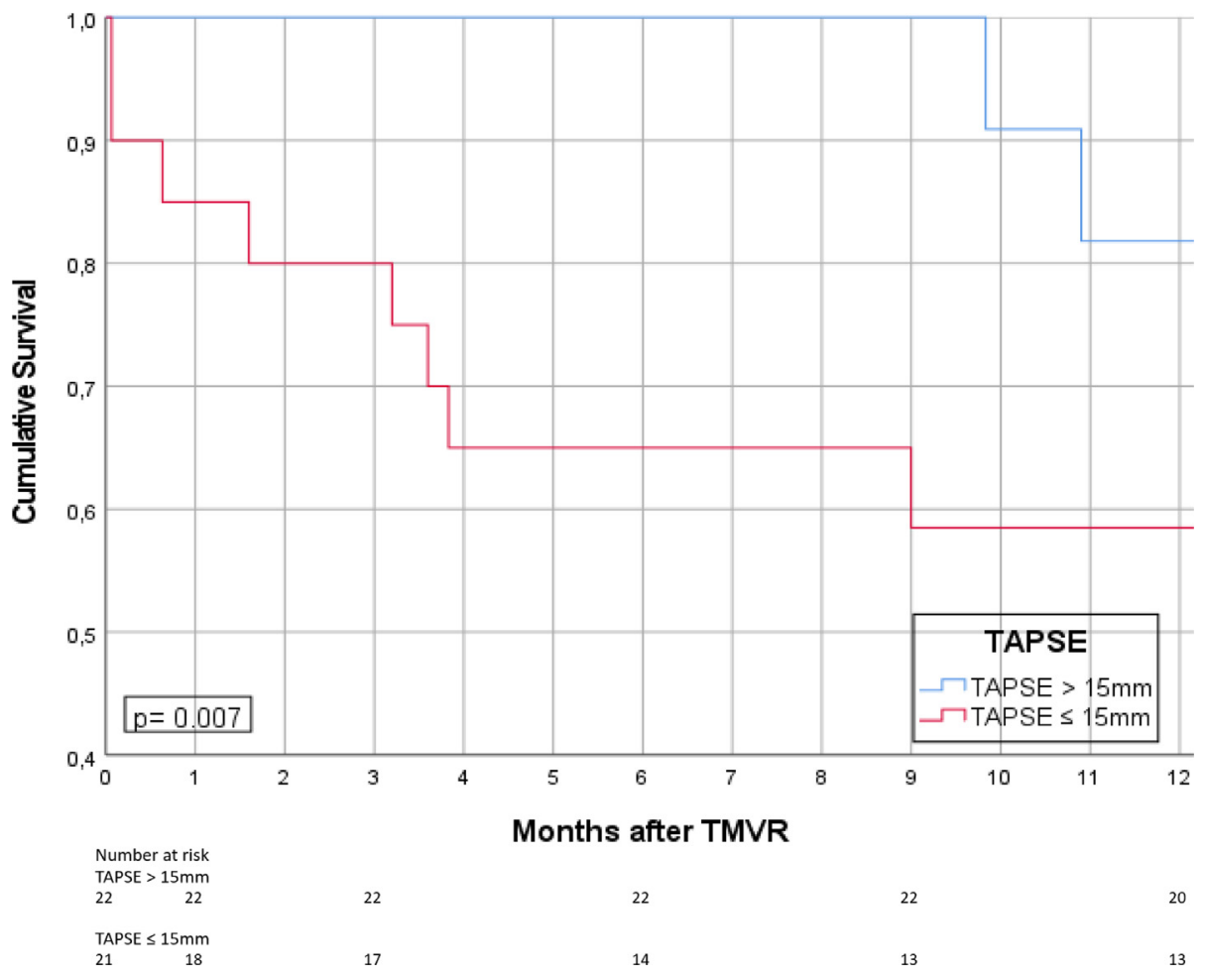

Fig. 2. Survival of FMR-patients with and without RVD after TMVR.

sents the receiver operating curves of the SHFM score and MAGGIC score in these patients.

Also in separate analysis of patients with MR of functional and degenerative origin, SHFM and MAGGIC score displayed corre- sponding AUROC values (FMR group: 0.696 for SHFM score and 0.722 for the MAGGIC score; DMR group: 0.727 for SHFM and 0.629 for the MAGGIC score). 


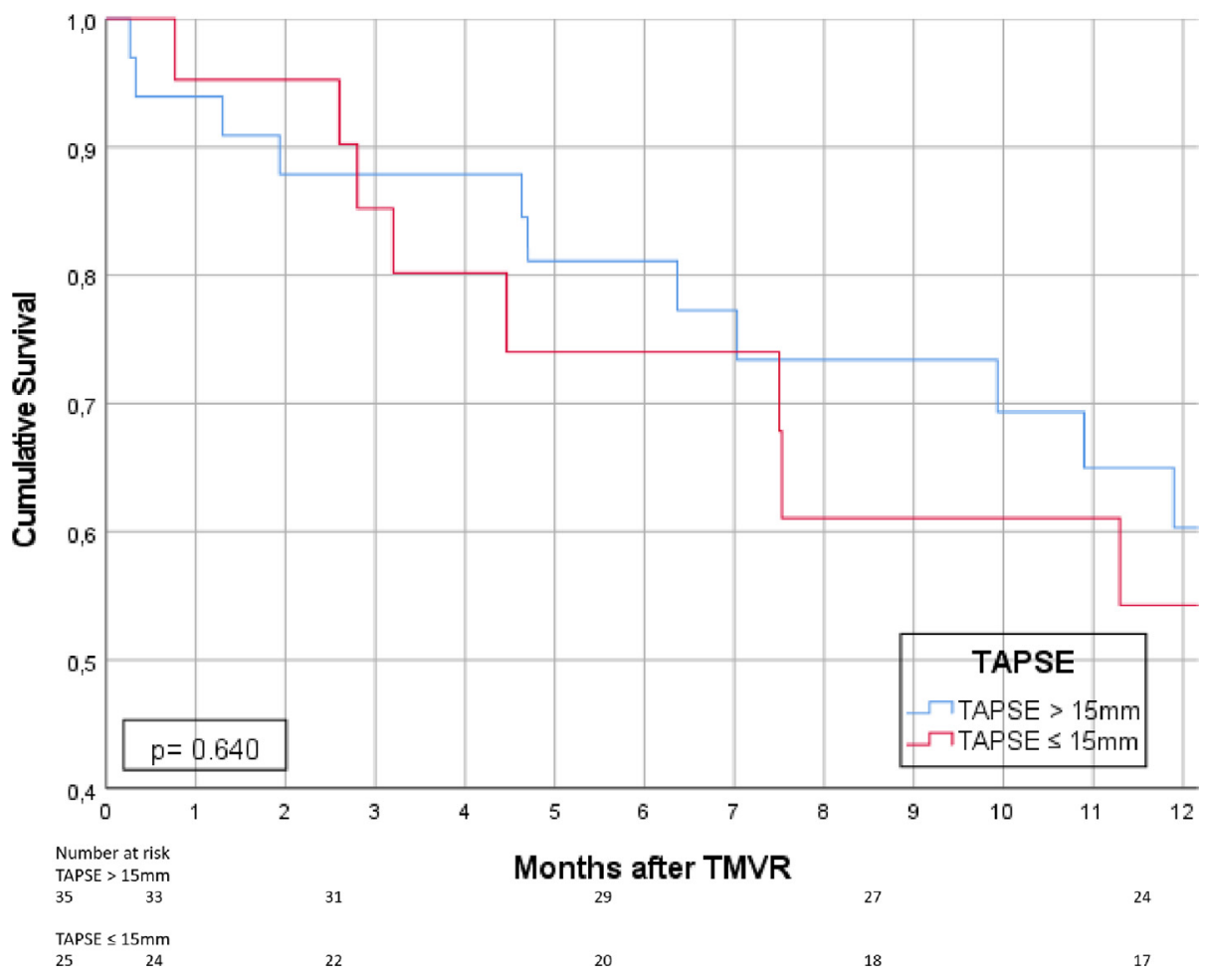

Fig. 3. Survival of DMR-patients with and without RVD after TMVR.

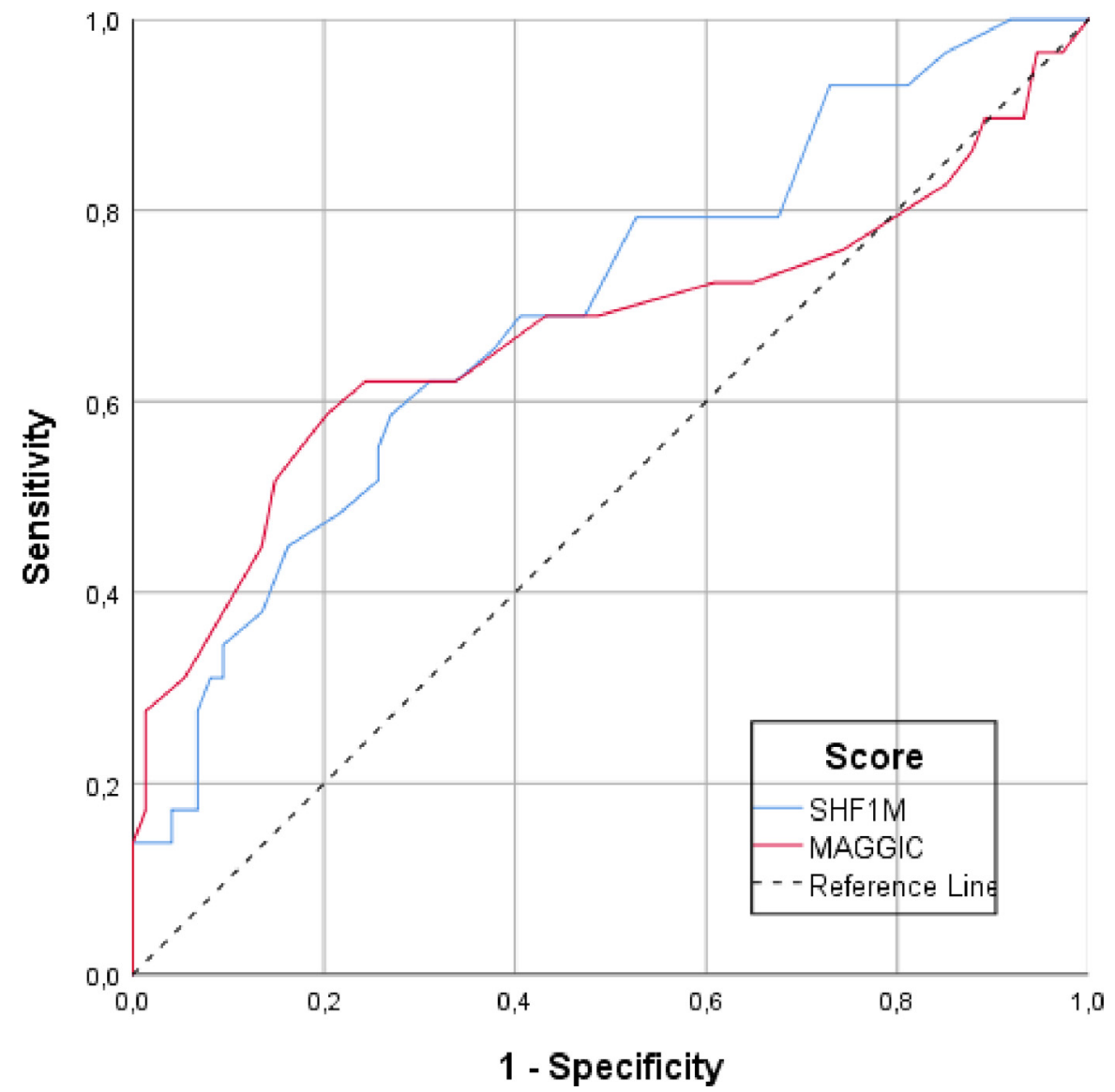

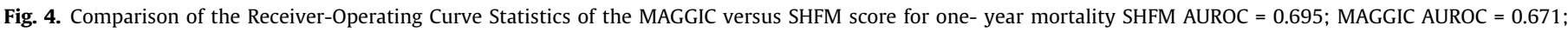
$P>0.05$, DeLong AUROC comparison. 
However, after separate analysis of the subsets of patients with preserved and reduced right ventricular function, SHFM and MAGGIC scores displayed differential prognostic performance:

In the subset of patients with RVD present at the time of TMVR, both scoring systems provided modest and comparable prognostic utility with an AUROC value of 0.665 for the SHFM score and 0.788 for the MAGGIC score, see Fig. 5.

In contrast, in patients with preserved right ventricular function at TMVR, SHFM score as well demonstrated an adequate AUROC value of 0.755 , whereas the MAGGIC score provided only poor prognostic utility in the subset of patients without RVD with an AUROC value of $0.511, \mathrm{p}=0.019$ (see Fig. 6).

By multivariate analysis including RVD as well as MAGGIC and SHFM score, only a high SHFM score persisted as independent predictor of one-year all-cause mortality in these patients with a hazard ratio of $1.03,95 \%$ confidence interval 1.013-1.046, $\mathrm{p}<0.001$.

\section{Discussion}

The present study investigates the prognostic utility of right ventricular dysfunction, the MAGGIC score and Seattle Heart Failure model in a non-selected all-comers population with severe mitral regurgitation of functional and degenerative origin after TMVR. Whereas both right ventricular dysfunction and the examined scoring systems represent validated tools for the prediction of survival in heart failure patients also after TMVR [17,29-31], their utility in the growing number of patients undergoing TMVR for severe MR has not been investigated comparatively so far. In

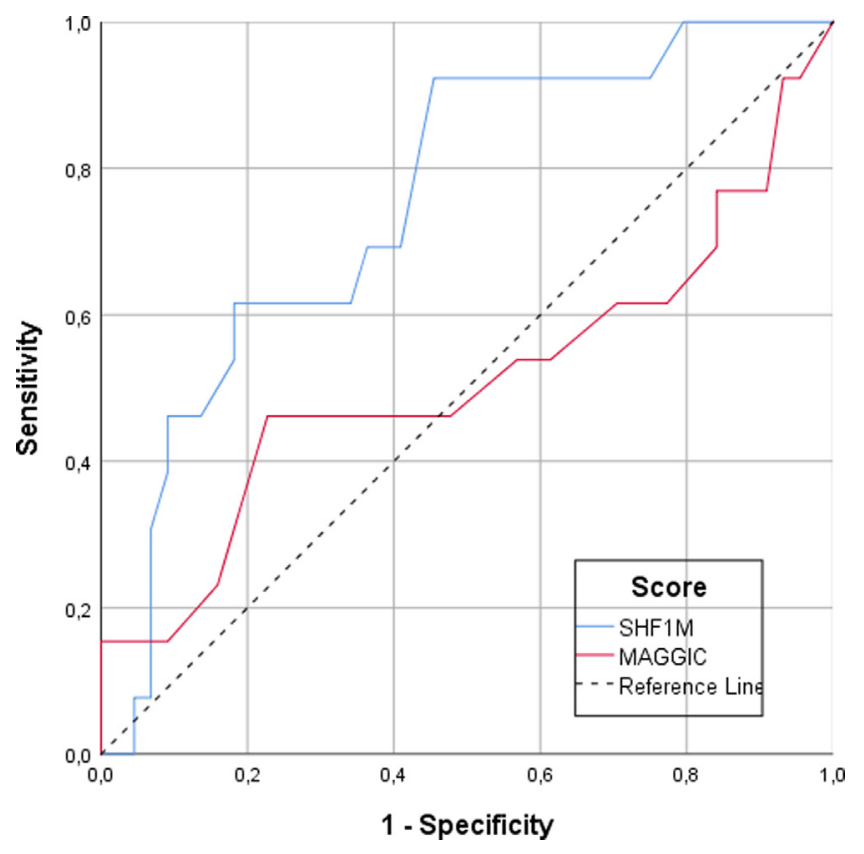

Fig. 6. Comparison of the Receiver-Operating Curve Statistics of the MAGGIC versus SHFM score for one-year mortality in patients without right ventricular dysfunction $($ RVD). SHFM AUROC $=0.755 ;$ MAGGIC AUROC $=0.511 ; P<0.019$, DeLong AUROC comparison.

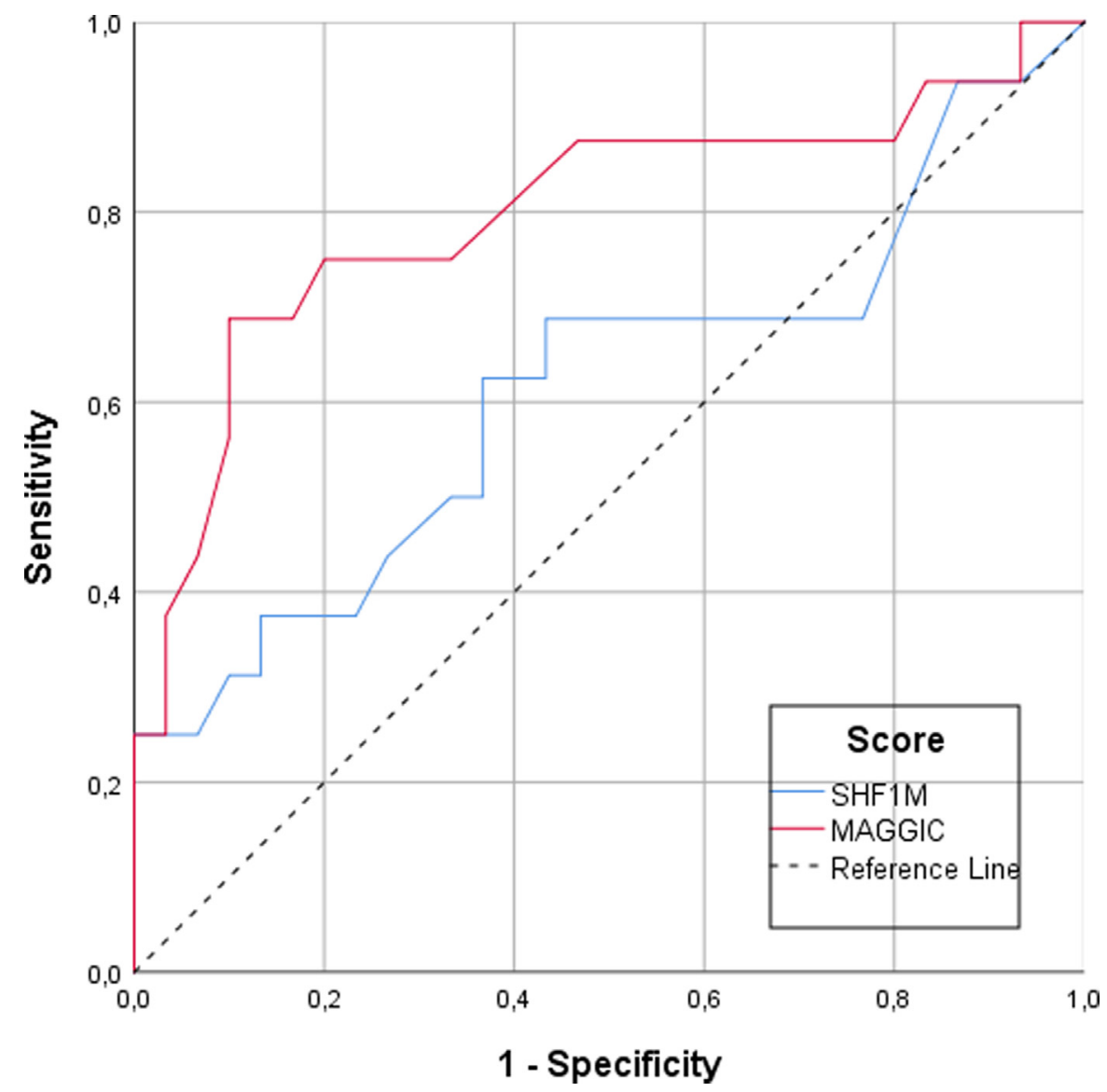

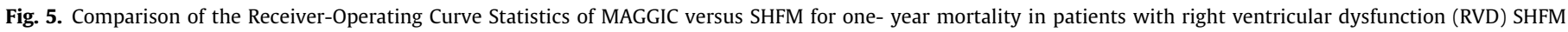
AUROC $=0.615 ;$ MAGGIC AUROC $=0.799 ; \mathrm{p}=>0.05$. 
recent large register reports, the impact of right ventricular failure has only been reflected indirectly by severe tricuspid insufficiency $[4,32]$.

One key finding of this study is that the negative prognostic impact of RVD on outcome in FMR-patients after TMVR can also be assessed by the easily obtainable measurement of TAPSE as described by Kaneko et al. [22] and is not dependent on the more complex tissue doppler imaging [33].

In general, echocardiographic assessment of right ventricular failure should comprise several parameters [34-36]. The present study investigates only one of them: TAPSE may be variable depending on different concomitant factors [37] and represents only the longitudinal right ventricular excursion with minor informative value of the free RV wall motion capacity. However, TAPSE showed good correlation with RVEF measured in radionuclide studies [21]. It has to be pointed out that a more detailed analysis of RV function might have augmented the prognostic value of RVD in this study.

Both heart failure scores examined in this study provide moderate overall sensitivity and specificity for one-year mortality in these patients. The application of scoring systems initially designed for other entities is common and feasible [17,38]. Our findings stand in good accordance to the paper of Schau et al. [17] regarding applicability of the SHFM and MAGGIC score also to MC patients.

However, as right heart failure has a well described negative prognostic impact also in TMVR-patients [22,33], we felt that the generalizability of these common heart failure scores in heterogenous groups of FMR and DMR with and without right heart failure should be confirmed separately. Indeed, the key finding of this study is that the prognostic value of the MAGGIC score is dependent on the presence or absence of RVD.

In contrast, the SHFM score provided moderate predictive power also in this subset of patients and persisted as independent significant predictor of adverse outcome in this study.

The interpretation of this finding appears complex. The SHFM and MAGGIC score are based on different parameters and different derivation cohorts, with a presumed minority of patients with severe MR. Further, we only can speculate that the populations used for developing the MAGGIC score with both HFrEF and HFpEF [27] mainly consisted of patients with concomitant RVD, whereas the SHFM patients included a broader spectrum of both right ventricular function and baseline parameters reflecting the patientś characteristics in this study more precisely.

A more detailed stratification of patients by SHFM level and RVD did not provide additional information (data not shown), most probably due to the limited number of data.

Our study bears a number of limitations to be considered when interpreting these findings:

The retrospective design of the study did not allow to calculate and consider further echocardiographic parameters like strain imaging. Due to the lack of complete follow ups, further associations of baseline right heart function and clinical effects could not be performed. The limited number of patients; including those first to be treated in this institution might explain the higher 1year mortality than expected from contemporary TMVR trials [7,9].

\section{Conclusion}

Both, right ventricular dysfunction defined by TAPSE and the Seattle Heart Failure model have predictive power for prediction of survival in patients with severe mitral regurgitation undergoing TMVR. Whereas TAPSE as a standardized and easily measurable echocardiographic parameter should be evaluated in every patient screened for TMVR, the more elaborate calculation of the SHFM score gives additional prognostic information irrespective of concomitant right heart dysfunction.

As a substantial number of heart failure patients develops advanced MR during the course of disease, our findings might contribute to a more personalized evaluation of prognosis and therapeutic approach in heart failure patients with MR undergoing TMVR.

\section{Declaration of Competing Interest}

The authors report no relationships that could be construed as a conflict of interest.

\section{References}

[1] T. Feldman et al., Percutaneous repair or surgery for mitral regurgitation, N. Engl. J. Med. 364 (15) (2011) 1395-1406.

[2] S.V. Arnold et al., Association of transcatheter mitral valve repair with quality of life outcomes at 30 Days and 1 Year: analysis of the transcatheter valve therapy registry, JAMA Cardiol. 3 (12) (2018) 1151-1159.

[3] D.D. Glower et al., Percutaneous mitral valve repair for mitral regurgitation in high-risk patients: results of the EVEREST II study, J. Am. Coll. Cardiol. 64 (2) (2014) 172-181.

[4] M. Puls et al., One-year outcomes and predictors of mortality after MitraClip therapy in contemporary clinical practice: results from the German transcatheter mitral valve interventions registry, Eur. Heart J. 37 (8) (2016) $703-712$.

[5] J. Cleland et al., Predicting the long-term effects of cardiac resynchronization therapy on mortality from baseline variables and the early response a report from the CARE-HF (Cardiac Resynchronization in Heart Failure) Trial, J. Am. Coll. Cardiol. 52 (6) (2008).

[6] M. Enriquez-Sarano, I. Thoralf, M. Sundt, Early Surgery Is Recommended for Mitral Regurgitation, 2010.

[7] G.W. Stone et al., Transcatheter mitral-valve repair in patients with heart failure, N. Engl. J. Med. 379 (24) (2018) 2307-2318.

[8] M. Mack, COAPT: Three-Year Outcomes from a Randomized Trial of Transcatheter Mitral Valve Leaflet Approximation in Patients with Heart Failure and Secondary Mitral Regurgitation, in: TCT, 2019. San Francisco.

[9] J.F. Obadia et al., Percutaneous repair or medical treatment for secondary mitral regurgitation, N. Engl. J. Med. 379 (24) (2018) 2297-2306.

[10] R. Al-Bawardy et al., Association of pulmonary hypertension with clinical outcomes of transcatheter mitral valve repair, JAMA Cardiol. (2019).

[11] S. Arora et al., The prevalence and impact of atrial fibrillation on 1-year outcomes in patients undergoing transcatheter mitral valve repair: results from the society of thoracic surgeons/American College of cardiology transcatheter valve therapy registry, JACC Cardiovasc. Interv. 12 (6) (2019) 569-578.

[12] J. Ledwoch et al., Prognostic impact of left atrial function following transcatheter mitral valve repair, J. Am. Heart Assoc. 8 (9) (2019) e011727.

[13] H. Hioki et al., The MAGGIC risk score predicts mortality in patients undergoing transcatheter aortic valve replacement: sub-analysis of the OCEAN-TAVI registry, Heart Vessels 34 (12) (2019) 1976-1983.

[14] S. Li et al., Validity of the Seattle Heart Failure Model after heart failure hospitalization, ESC Heart Fail. 6 (3) (2019) 509-515.

[15] A.P. Kalogeropoulos et al., Utility of the Seattle Heart Failure Model in patients with advanced heart failure, J. Am. Coll. Cardiol. 53 (4) (2009) 334-342.

[16] J. Honold et al., Comparison of the Seattle heart failure model and cardiopulmonary exercise capacity for prediction of death in patients with chronic ischemic heart failure and intracoronary progenitor cell application, Clin. Cardiol. 36 (3) (2013) 153-159.

[17] T. Schau et al., Long-term survival after MitraClip $((\mathrm{R}))$ therapy in patients with severe mitral regurgitation and severe congestive heart failure: a comparison among survivals predicted by heart failure models, J. Cardiol. 67 (3) (2016) 287-294.

[18] S.A.M. Nashef et al., EuroSCORE II, Eur. J. Cardiothorac. Surg. 41 (4) (2020) $734-$ 745.

[19] H. Baumgartner et al., 2017 ESC/EACTS Guidelines for the management of valvular heart disease, Eur. Heart J. 38 (36) (2017) 2739-2791.

[20] F. Ferrara et al., Physiologic correlates of tricuspid annular plane systolic excursion in 1168 healthy subjects, Int. J. Cardiol. 223 (2016) 736-743.

[21] O. Ueti et al., Assessment of Right Ventricular Function With Doppler Echocardiographic Indices Derived From Tricuspid Annular Motion: Comparison With Radionuclide Angiography, Heart (Br. Cardiac Soc.) 88 (3) (2002).

[22] H. Kaneko et al., Prognostic significance of right ventricular dysfunction in patients with functional mitral regurgitation undergoing MitraClip, Am. J. Cardiol. 118 (11) (2016).

[23] P.G. Yock, R.L. Popp, Noninvasive estimation of right ventricular systolic pressure by Doppler ultrasound in patients with tricuspid regurgitation, Circulation 70 (4) (1984) 657-662. 
[24] C. Levy Wayne et al., The seattle heart failure model, Circulation 113 (11) (2006) 1424-1433.

[25] D. Mozaffarian et al., Prediction of mode of death in heart failure: the Seattle Heart Failure Model, Circulation 116 (4) (2007) 392-398.

[26] W.C. Levy et al., Maximizing survival benefit with primary prevention implantable cardioverter-defibrillator therapy in a heart failure population, Circulation 120 (10) (2009) 835-842.

[27] S.J. Pocock et al., Predicting survival in heart failure: a risk score based on 39 372 patients from 30 studies, Eur. Heart J. 34 (19) (2013) 1404-1413.

[28] P. Ponikowski et al., 2016 ESC Guidelines for the diagnosis and treatment of acute and chronic heart failure: The Task Force for the diagnosis and treatment of acute and chronic heart failure of the European Society of Cardiology (ESC) Developed with the special contribution of the Heart Failure Association (HFA) of the ESC, Eur. Heart J. 37 (27) (2016) 2129-2200.

[29] S. Ghio et al., Prognostic usefulness of the tricuspid annular plane systolic excursion in patients with congestive heart failure secondary to idiopathic or ischemic dilated cardiomyopathy, Am. J. Cardiol. 85 (7) (2000).

[30] J. Polak et al., Right ventricular ejection fraction: an indicator of increased mortality in patients with congestive heart failure associated with coronary artery disease, J. Am. Coll. Cardiol. 2 (2) (1983).

[31] P. de Groote et al., Right ventricular ejection fraction is an independent predictor of survival in patients with moderate heart failure, J. Am. Coll. Cardiol. 32 (4) (1998).
[32] D. Kalbacher et al., Long-term outcome, survival and predictors of mortality after mitraclip therapy: results from the German Transcatheter Mitral Valve Interventions (TRAMI) Registry, Int. J. Cardiol. 277 (2019).

[33] C. Giannini et al., Right ventricular evaluation to improve survival outcome in patients with severe functional mitral regurgitation and advanced heart failure undergoing MitraClip therapy, Int. J. Cardiol. 223 (2016).

[34] V. Harjola et al., Contemporary management of acute right ventricular failure: a statement from the heart failure association and the working group on pulmonary circulation and right ventricular function of the european society of cardiology, Eur. J. Heart Fail. 18 (3) (2016).

[35] R. Lang et al., Recommendations for cardiac chamber quantification by echocardiography in adults: an update from the American Society of echocardiography and the European association of cardiovascular imaging, J. Am. Soc. Echocardiogr.: Off. Publ. Am. Soc. Echocardiogr. 28 (1) (2015).

[36] S. Kaul et al., Assessment of right ventricular function using two-dimensional echocardiography, Am. Heart J. 107 (3) (1984).

[37] S. Hsiao et al., Severe tricuspid regurgitation shows significant impact in the relationship among peak systolic tricuspid annular velocity, tricuspid annular plane systolic excursion, and right ventricular ejection fraction, J. Am. Soc. Echocardiogr.: Off. Publ. Am. Soc. Echocardiogr. 19 (7) (2006).

[38] T. Ando et al., The clinical value of the PRECISE-DAPT score in predicting longterm prognosis in patients with acute myocardial infarction, Int. J. Cardiol. Heart Vasc. 29 (2020) 100552. 